\title{
Ultrabroadband microwave radiation from near- and mid-infrared laser-produced plasmas in air
}

\author{
Alexander Englesbe $\odot,{ }^{1, *}$ Jennifer Elle, ${ }^{2}$ Robert Schwartz $\odot,{ }^{3}$ Travis Garrett, ${ }^{2}$ Daniel Woodbury $\odot,{ }^{3}$ Dogeun Jang $\odot,{ }^{3}$ \\ Ki-Yong Kim, ${ }^{3}$ Howard Milchberg ${ }^{\circ},{ }^{3}$ Remington Reid, ${ }^{2}$ Adrian Lucero, ${ }^{2}$ Daniel Gordon, ${ }^{1}$ Ryan Phillips, ${ }^{2}$ \\ Serge Kalmykov $\mathbb{1}^{2,4}$ and Andreas Schmitt-Sody $\mathbb{1}^{2}$ \\ ${ }^{1}$ Plasma Physics Division, Naval Research Laboratory, Washington, DC 20375, USA \\ ${ }^{2}$ High Power Electromagnetics Division, Air Force Research Laboratory, Kirtland AFB, New Mexico 87117, USA \\ ${ }^{3}$ Institute for Research in Electronics and Applied Physics, University of Maryland, College Park, Maryland 20742, USA \\ ${ }^{4}$ Leidos Innovations Center, Albuquerque, New Mexico 87106, USA
}

(Received 19 January 2021; revised 28 May 2021; accepted 8 June 2021; published 16 July 2021)

\begin{abstract}
An ultrashort laser pulse focused in air creates a plasma that radiates broadband electromagnetic waves. We experimentally compare the generation of microwaves from plasmas produced with two different laser systems that operate in the near- and mid-infrared regimes. Changing the laser wavelength increases the microwave power by 100 times and changing the input pulse energy allows for tuning of the microwave frequency spectrum, which we absolutely calibrate over a range of $2-70 \mathrm{GHz}$. The variation of the spectrum with laser pulse energy confirms the existence of a distinct mechanism that generates microwave radiation from laser-produced plasmas in gases. We propose that a radial diffusive expansion wave of the plasma electrons drives a longitudinal current along the plasma surface whose amplitude varies with the total residual electron energy imparted by the laser field and this longitudinal current produces the detected radiation.
\end{abstract}

DOI: 10.1103/PhysRevA.104.013107

\section{INTRODUCTION}

Ultrashort pulse laser-produced plasmas are versatile sources of secondary radiation, that is, electromagnetic radiation arising from currents driven in the plasma by the laser pulse. For a laser pulse focused in gas, there are many possible mechanisms that have been closely studied that can cause emission above and below the laser frequency [1-5]. Low-frequency secondary radiation at terahertz $(\mathrm{THz})$ frequencies from plasmas generated in air has been investigated extensively with near-infrared (NIR) laser systems typically using two-color pulses [6,7] and has more recently been measured using mid-infrared (MIR) laser drivers [8,9]. However, these plasmas also radiate at even lower frequencies in the microwave, that is, the gigahertz $(\mathrm{GHz})$ regime [10-14]. The mechanism that causes emission on nanosecond timescales, which are much longer than the laser pulse duration $(\sim 100 \mathrm{fs})$ or plasma frequency $\left(2 \pi / \omega_{p} \sim 1 \mathrm{ps}\right)$, has not been identified by prior investigations.

This paper reports the results of a systematic experimental study of the microwave generation from air plasma in the spectral range of 2 to $70 \mathrm{GHz}$. The plasma emitting the microwaves is created by optical field ionization with NIR $\left(\lambda_{0}=\right.$ $0.8 \mu \mathrm{m})$ or $\operatorname{MIR}\left(\lambda_{0}=3.9 \mu \mathrm{m}\right)$ pulses focused in ambient air.

\footnotetext{
*Corresponding author: alexander.englesbe@nrl.navy.mil

Published by the American Physical Society under the terms of the Creative Commons Attribution 4.0 International license. Further distribution of this work must maintain attribution to the author(s) and the published article's title, journal citation, and DOI.
}

The experiments accomplish two purposes. First, we show that the microwave amplitude and frequency may be tuned by changing the laser wavelength and pulse energy, respectively. We measure a 100 -fold increase in the emitted microwave power merely by increasing the laser wavelength while keeping the focusing optics and laser pulse energy the same. When we increase the laser energy at both wavelengths, we find that the peak microwave frequency decreases. Second, these results prove that the microwaves arise from a distinct generation mechanism that is supported by simulations of the ionization caused by the laser propagation.

As the laser pulse passes, the electrons near the boundary of the plasma column produced by the intense field expand radially outward in a conical shell due to their residual energy. The residual energy is the electron energy distribution imparted by the laser field to the electron population [15-18]. The highest-energy component of the distribution scales with laser intensity and wavelength like $I_{0} \lambda_{0}^{2}$ (for further discussion see Sec. 1 in the Supplemental Material [19]). The conical expansion of the electrons evolves over a timescale on the order of $100 \mathrm{ps}$ (which corresponds to the microwave frequencies we measure) and its asymmetry in the direction of the laser propagation stimulates a longitudinal surface current on the outer boundary of the finite conductivity plasma column. This surface current travels coherently behind the ionizing laser pulse, thereby building up a radially polarized electromagnetic wave. The wave then detaches from the end of the plasma column and propagates into the far field as a broadband microwave pulse.

Pulsed microwaves with bandwidth as large as we have observed in these experiments can usually only be achieved with microwave photonic devices (see, for example, [20]). 


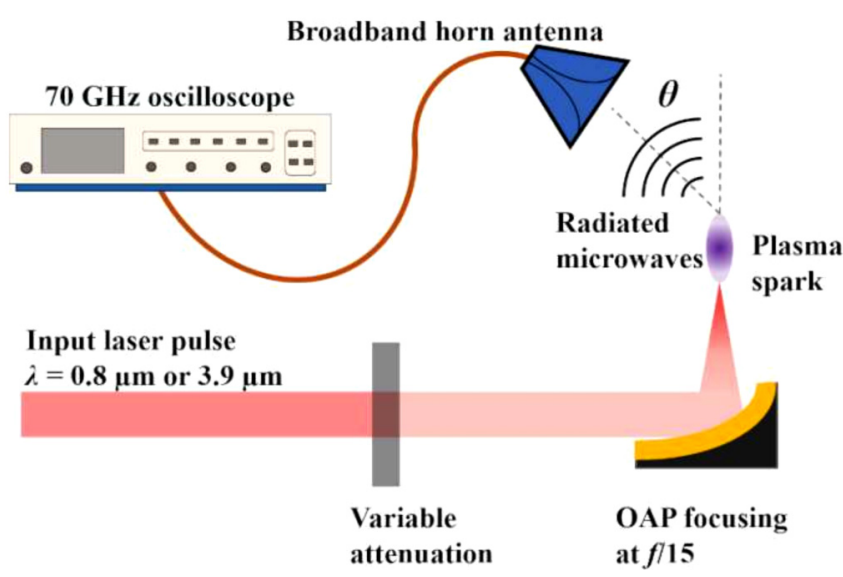

FIG. 1. Experimental setup for measuring microwave radiation from the laser sparks in air. The horn antenna translates angularly around the plasma at a fixed radius. An angular antenna position of $\theta=0^{\circ}$ corresponds to the laser axis.

Creating them by other means involves complicated microwave circuit design techniques such as a photoconductive resonator [21], a pulse forming network that shortens a longer duration pulse [22], or a specialized monolithic microwave integrated circuit [23]. The plasmas are able to provide comparable or greater bandwidth and a large tuning range simply by tightly focusing high-intensity femtosecond laser pulses in air.

To date, experimental observations of radio-frequency and microwave radiation from laser plasmas are usually associated with the interaction of nanosecond to femtosecond pulses with solids (see, for example, Refs. [24-27]). We find the cause of the microwave radiation from the air plasma to be physically dissimilar from that in laser-solid interactions, although in both cases the source currents can be considered to arise from the high-energy tails of the respective electron energy distributions in the laser-produced plasmas [28,29].

\section{EXPERIMENTAL METHODS}

The MIR laser in our experiments uses staged optical parametric chirped pulse amplification to produce ultrashort pulses centered at $\lambda_{0}=3.9 \mu \mathrm{m}$ with a duration of approximately $100 \mathrm{fs}$ at a repetition rate of $20 \mathrm{~Hz}$ [30]. The NIR experiments are performed on a traditional Ti:sapphire chirped pulse amplification system with $\lambda_{0}=0.8 \mu \mathrm{m}$ and a 50 -fs pulse duration operating at $10 \mathrm{~Hz}$. The uncertainty in the pulse durations for both lasers is about $10 \%$. Figure 1 shows the setup for both the NIR and MIR microwave generation experiments.

A diaphragm iris at the laser outputs limits their apertures to a $10 \mathrm{~mm}$ diameter. The iris provides a circular shape to the transverse profile of the MIR laser so that the laser conditions are closer to those of the NIR laser. The apertured MIR pulses have some transverse structure, which will decrease the maximum focal intensity relative to an ideal Gaussian beam. The energy of the input pulses at $\lambda_{0}=0.8$ and $3.9 \mu \mathrm{m}$ is set within a range from 2 to $12 \mathrm{~mJ}$, with a typical shot-to-shot fluctuation of $10 \%$. An off-axis parabolic (OAP) mirror with a 150 -mm reflected focal length focuses the pulses at $f / 15$ to make a plasma spark that is less than or equal to $1 \mathrm{~cm}$ in length. While the f-number and input pulse energies are standardized between the two laser systems, it is necessary to shorten the pulse duration of the NIR laser relative to the MIR laser in order to produce measurable signal levels from the NIR laser plasma. This is why the NIR laser pulse duration is $50 \mathrm{fs}$, whereas it is $100 \mathrm{fs}$ for the MIR laser.

Our experimental setup is sufficient to demonstrate that the laser wavelength can significantly influence the production of the microwave radiation. However, to experimentally measure the precise scaling of the microwave power with laser wavelength and intensity as it relates to the electron residual energy, one would have to account for the wavelength dependence of the beam waist and the resulting plasma diameter by carefully controlling the characteristics of the input laser pulses. Nonetheless, our simulations of the laser propagation in these experiments show that the beam waist and plasma diameter are largely similar for the MIR and NIR laser pulses (the simulations assume ideal input beam quality). It is important to note that since we have used a 10 -mm-diameter beam incident on a $150-\mathrm{mm}$ focal length OAP mirror at both values of $\lambda_{0}$, the ideal vacuum spot diameter and therefore intensity through the focal region have wavelength dependence. For a Gaussian transverse intensity profile, the Rayleigh range and focused spot diameter in vacuum both scale with $\lambda_{0}$ and therefore the intensity scales with $\lambda_{0}^{-2}$. In atmosphere, plasma-induced defocusing and nonlinear self-focusing due to the Kerr effect determine the focal intensity and spot size. These effects are accounted for in the laser propagation simulations.

The bandwidth of the microwave pulses radiated from the air plasmas is so broad that it is impractical to measure the radiation in a single laser shot. We use four standard broadband horn antennas to cover the complete frequency range from 2 to $70 \mathrm{GHz}$. A Tektronix DPO77002SX with 70-GHz real-time bandwidth digitizes the microwave waveforms. The oscilloscope sets the upper frequency limit of our measurements, and each horn antenna operates in a subrange of its total bandwidth $(2-18,18-40,40-60$, and 50-70 GHz). The spectral and waveform amplitude measurements are averaged from hundreds of laser shots.

Each antenna is mounted on a rotating gantry at a fixed distance of $55 \mathrm{~cm}$ from the plasma, which is the maximum achievable given the space constraints in the laboratory. Spectral-angular maps of the microwaves are recorded using both laser systems by translating each antenna about the plasma in $10^{\circ}$ increments from close to the laser axis at $\theta \simeq 0^{\circ}$ to its normal at $90^{\circ}$. Reflections of the microwaves off any nearby surfaces are either damped with microwave absorbing foam or discriminated by time of flight since the pulse duration is very short. The antennas are used in succession in an attempt to keep constant the orientation between their apertures and the plasma at each observation angle. Our measurements rely on the high shot-to-shot repeatability of the microwave radiation. The shot-to-shot variance in the microwave waveform amplitude is determined by the fluctuation in the laser pulse energy.

There is a unique response function for each antenna and the coaxial cable connecting it to the oscilloscope. The spectrum from each must be absolutely calibrated in order 

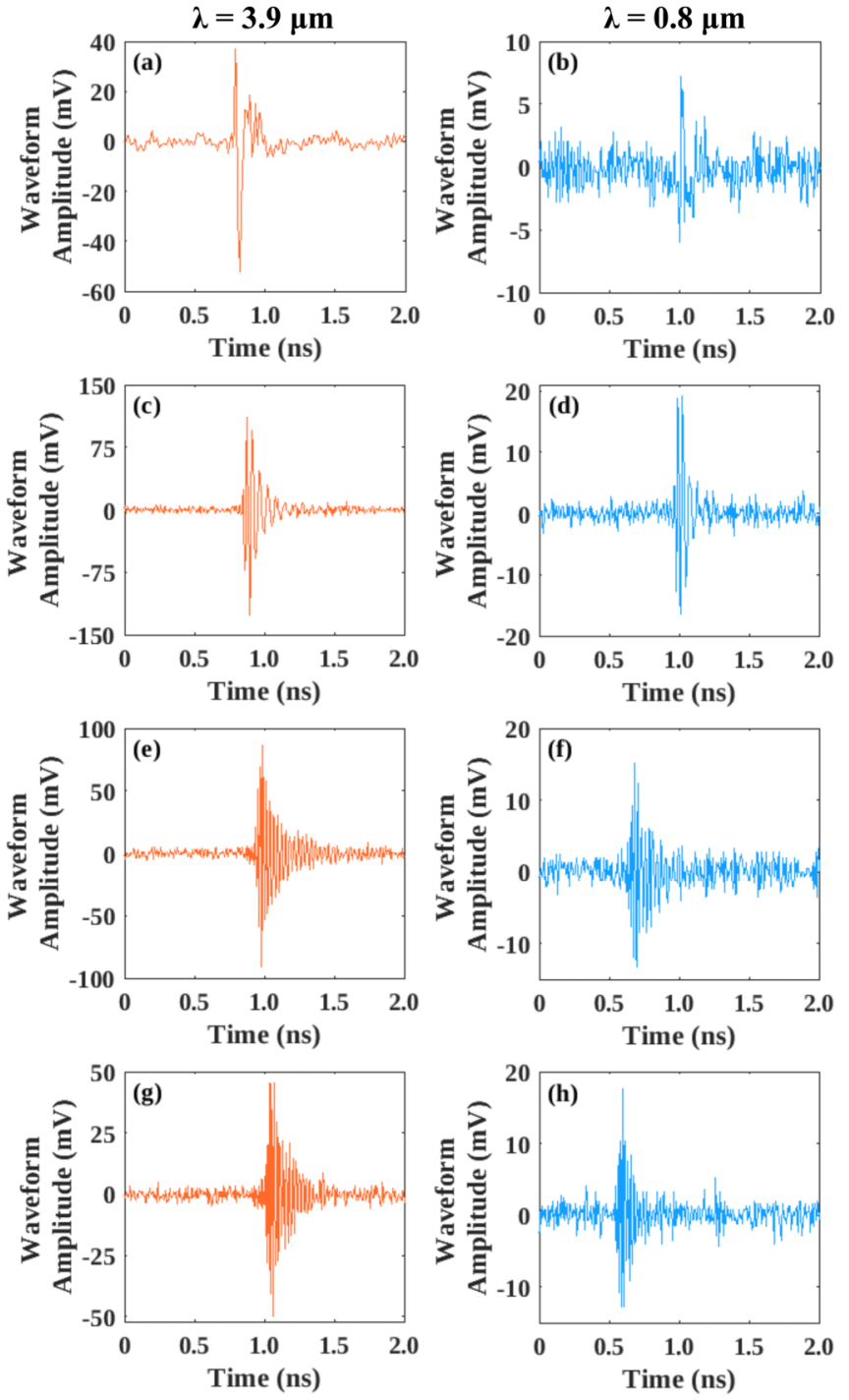

FIG. 2. Time domain voltage waveforms of the microwave fields radiating from the air plasmas at a constant laser pulse energy of $12 \mathrm{~mJ}$. The fields are received with antennas operating at (a) and (b) 2-18 GHz, (c) and (d) 18-40 GHz, (e) and (f) 40-60 GHz, and (g) and (h) 50-70 GHz for (a), (c), (e), and (g) $\lambda_{0}=3.9 \mu \mathrm{m}$ and (b), (d), (f), and (h) $\lambda_{0}=0.8 \mu \mathrm{m}$. The horns are located at $\theta=30^{\circ}$ off the laser axis. The time axis in each plot is based on an arbitrary reference for time $t=0$, as the trigger signal cable lengths are different for both of the lasers, and the microwave cables used with each of the horns also do not all have the same length.

to present spectra as if they were measured with a single instrument having uniform frequency response. We use reference noise sources that produce known microwave power spectral densities to measure the real part of the response function to calculate calibration factors for each coaxial cable with the oscilloscope. The four antennas are calibrated separately using the two antenna technique [31] to measure their gain as a function of frequency. We do not measure the full complex transfer functions, so we cannot combine the different antenna waveforms in the time domain, which is why Figs. 2 and 3 use uncalibrated data. Further details describing how to perform the calibrations are given in Sec. 2 in [19].

\section{RESULTS}

\section{A. Waveforms and amplitudes in the time domain}

We find a signal over the entire frequency range accessible to the four horn antennas we used to collect the radiation. For a single laser pulse energy and antenna observation angle, Fig. 2 shows the time domain voltage waveforms with each of the antennas. There is a measurable few-cycle microwave pulse in every case whose duration is about 500 ps. The waveforms appear to be negatively chirped. Horn antennas are dispersive, having complicated frequency-dependent phase responses [32] (over the broad frequency ranges the coaxial cables will also contribute dispersion) which likely cause the apparent chirp and the multiple oscillations of the waveforms. That is, the instrument response given by each combination of antenna and coaxial cable with the oscilloscope significantly influences the detailed structures of the waveforms in Fig. 2.

One main result of the experiments is the large amplitude difference between the MIR and NIR laser-produced microwaves. The amplitude difference can be found at any emission angle from the plasma, as shown in Fig. 3, which compares the waveform peak-to-peak voltage $V_{p p}$ as a function of the angular position of the antennas $\theta$ at a fixed laser energy of $2 \mathrm{~mJ}$. Direct comparison of the time domain amplitudes between the cases of laser wavelength is only valid for an individual antenna and accompanying coaxial cable (as opposed to comparing, for example, $V_{p p}$ for $2-18 \mathrm{GHz}$ with that for 50-70 GHz). The order of magnitude increase in $V_{p p}$ is observed over the range of emission angles and antenna frequency ranges. Here $V_{p p}$ is proportional to the microwave electric field amplitude and therefore $V_{p p}^{2}$ is proportional to the microwave power. This means that the MIR laser-produced plasma radiates on the order of 100 times the total microwave power of the plasma generated by the NIR laser.

Another characteristic to note is the shape of the angular distributions. The radiation is cylindrically symmetric about the laser propagation axis, even though Fig. 3 plots the angular dependence of $V_{p p}$ in the plane formed by the microwave electric field vector and the laser propagation direction. Figures 3(b)-3(d) indicate that the microwaves radiate in a forward-directed cone, with the angle of maximum emission occurring between the laser propagation axis $\left(\theta=0^{\circ}\right)$ and its normal $\left(\theta=90^{\circ}\right)$. There is some frequency dependence to the peak emission angle. Comparison of Figs. 3(b) and 3(d), for example, shows that while the peak emission angle in the $18-40 \mathrm{GHz}$ frequency range is $\theta \sim 70^{\circ}$, it is $\theta \sim 50^{\circ}$ in the $50-70 \mathrm{GHz}$ range. The conical radiation pattern whose maximum becomes more forward directed at higher frequencies implies that the current that produces the radiation flows parallel to the laser propagation axis and that it has a phase velocity approaching the group velocity of the laser pulse in the plasma. In general, a conical emission pattern with a null on axis is found for an electric dipole accelerating parallel to its dipole moment [33].

The polarization of the microwave electric field is also consistent with a longitudinal plasma current. In a cylindrical coordinate system, a longitudinal current produces an azimuthal magnetic field, which means that the electric field is radially polarized. We observe horizontal polarization of 
(a)

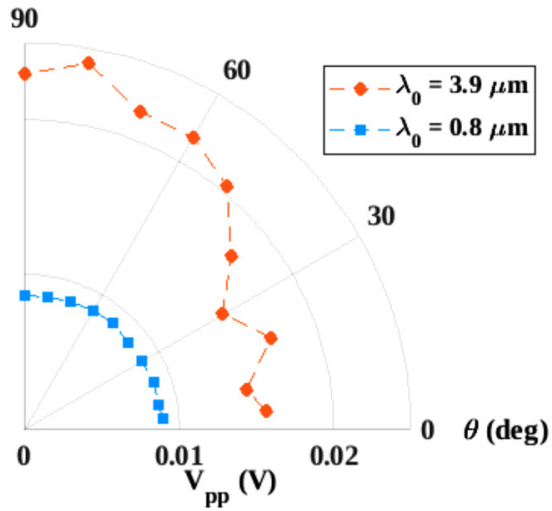

(c)

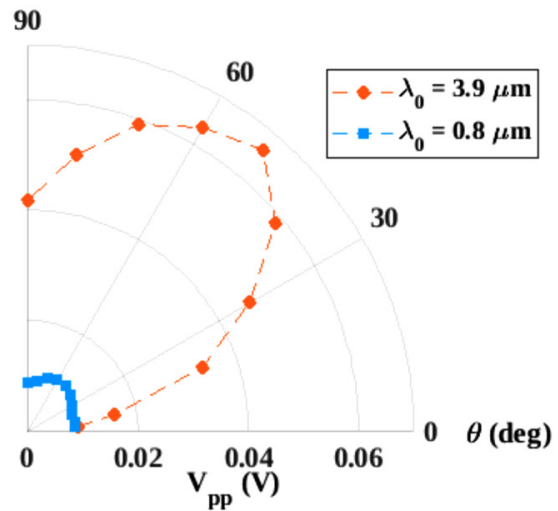

(b)

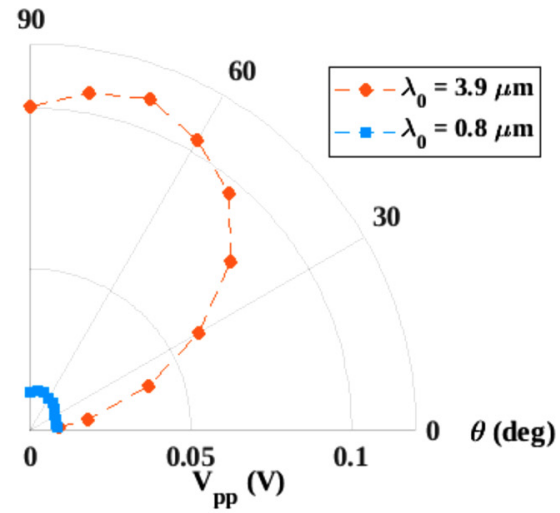

(d)

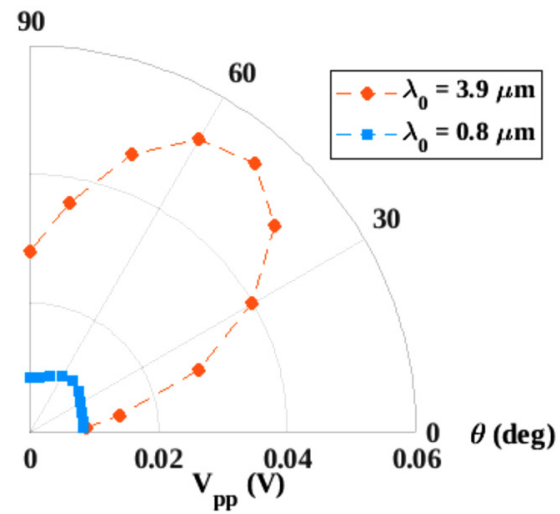

FIG. 3. Polar plots of the angular pattern of the radiation based on $V_{p p}$ for voltage waveforms recorded with the (a) 2-18 GHz, (b) $18-$ $40 \mathrm{GHz}$, (c) 40-60 GHz, and (d) 50-70 GHz antennas. The orange circles and blue squares correspond to $\lambda_{0}=3.9$ and $0.8 \mu \mathrm{m}$, respectively, at a laser pulse energy of $2 \mathrm{~mJ}$. The laser propagates from left to right.

the microwaves in the plane of the measurements and vertical polarization at an azimuthal position displaced $90^{\circ}$ from the measurement plane. The antennas are linearly polarized, so the signal amplitude is maximized when the angular difference between the incoming electric field vector and the antenna polarization (its $E$ plane) is minimized. We check for the microwave polarization by rotating the apertures of the horn antennas by $90^{\circ}$, similar to how one would use a linear polarizer to observe the polarization of optical light.

\section{B. Laser pulse energy dependence of the microwave power}

For both laser wavelengths, the radiated microwave power as a function of the input laser pulse energy increases at roughly the same rate over the range we used in the experiments. Instead of presenting measurements of $V_{p p}$, Fig. 4 shows the relative change in the total power integrated from 2 to $70 \mathrm{GHz}$ radiated per steradian as a function of laser energy. The measurement is repeated at three different observation angles. The total power is found by applying the calibration factors to the uncalibrated frequency spectra to get the absolute electric field spectrum, squaring it, and then integrating the result, which is a quantity proportional to the radiated power $\left(\int E^{2} d f \propto d P / d \Omega\right.$, where $E$ is spectral field amplitude, $P$ is power, and $\Omega$ is solid angle). The data in each plot are normalized to the power of the MIR-produced microwaves at a laser energy of $12 \mathrm{~mJ}$.

Figures 2-4 show different, complementary measurements to prove that the total microwave power radiated from the
MIR laser plasma is two orders of magnitude greater than that due to the NIR laser plasma. While the power also increases with input laser pulse energy, it appears that the wavelength dependence is stronger.

Increasing the laser pulse energy by a factor of 6 (from 2 to $12 \mathrm{~mJ}$ ) results in a similar increase in the total power. However, at a given laser pulse energy, increasing the wavelength by a factor of 5 results in 100 times the total power. The pulse energy and wavelength dependence we measure reflect the $I_{0} \lambda_{0}^{2}$ scaling of the electron residual energy, although other laser intensity and wavelength-dependent factors such as the plasma volume also play a role in determining the microwave power.

\section{Absolutely calibrated microwave frequency spectra}

Figure 5 shows the absolutely calibrated microwave spectra as a function of laser pulse energy. The spectra are the electric field amplitude in free space at the location of the antenna aperture. Their bandwidth is typically greater than $100 \%$, although each one contains a clear frequency peak. Observing the peaks would not be possible without calibrating the receivers. They have inverse dependence on the laser energy for both $\lambda_{0}=3.9$ and $0.8 \mu \mathrm{m}$. Therefore, the laser energy is also a means for tuning the microwave frequency content in these experiments.

The inverse laser pulse energy dependence of the frequency peaks proves that the microwaves arise from a distinct generation mechanism. We hypothesize that the peak frequency is 

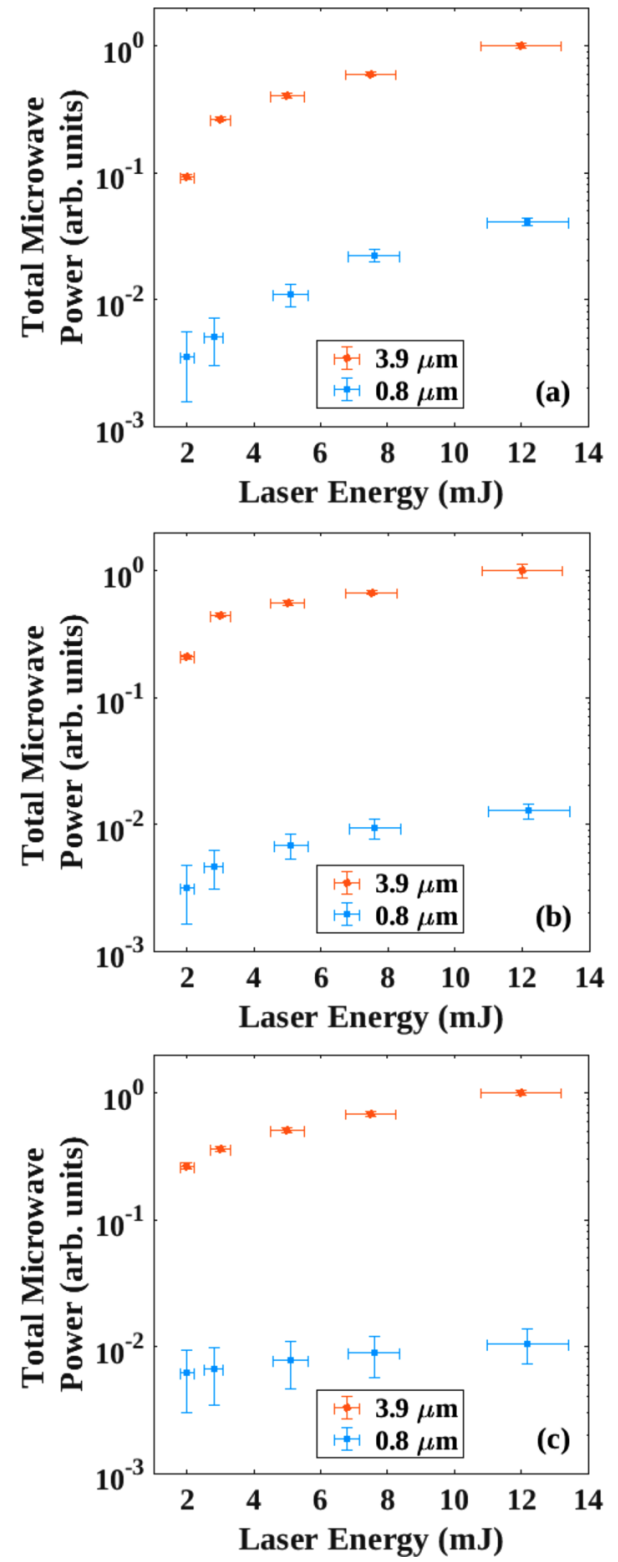

FIG. 4. Normalized total power integrated from 2 to $70 \mathrm{GHz}$ at each value of laser pulse energy at both laser wavelengths. The position of the antennas in each set of measurements is (a) $\theta=30^{\circ}$, (b) $\theta=60^{\circ}$, and (c) $\theta=90^{\circ}$. The error bars are calculated by finding the standard deviation of each electric field frequency component, adding these in quadrature since each normalized total power data point represents a numerical integration, and dividing by the number of frequency components in the spectrum.

related to the length of the laser plasma, which increases with the laser pulse energy. For the focusing f-number and laser energies we have used, the frequency spectra reach their peak in the range of tens of gigahertz.

Existing models for $\mathrm{THz}$ radiation from laser plasmas in air predict that its spectrum peaks in the $\mathrm{THz}$ at the plasma frequency [14,34-37]. They assume longitudinal charge separation in the core of the plasma driven by inverse bremsstrahlung and the ponderomotive force. The photocurrent model is predicated upon a two-color laser driver [4] and is not predicted to produce significant microwave radiation at atmospheric pressure [38]. Our experiments only involve single color pulses at the lasers' fundamental wavelengths and the results are not accounted for by the predictions of existing models.

The microwave generation should be considered an effect that is independent of the THz. In addition to the fact that the peaks we have measured are at much lower frequencies, the plasma frequency scales with the square root of the electron density. The maximum electron density is determined by the ionization rate, which has a strong dependence on the magnitude of the laser electric field. If the microwave frequency content were determined by the plasma frequency as is the case for the $\mathrm{THz}$, we would observe the opposite trend for the peaks with increasing laser energy in Fig. 5. Further, if the microwaves we measure were simply the low-frequency tail of the $\mathrm{THz}$ spectrum, then we would expect to observe in the microwave range a continuous spectrum increasing in amplitude up to the frequency limit of our receivers and not the spectral peaks that we did find. Therefore, the microwave radiation mechanism must be different from that which produces $\mathrm{THz}$ radiation often observed from air plasmas made with ultrashort laser pulses.

\section{DISCUSSION}

\section{A. Microwave generation mechanism}

We propose that the microwave radiation is due to the time asymmetry of the radial expansion of the outer shell of electrons from the plasma column. Owing to their residual energy, the electrons begin diffusive expansion out into the surrounding neutral atmosphere as soon as they are ionized. In atmosphere, elastic electron collisions with neutrals and ions moderate the expansion. By time asymmetry, we mean that the older electrons at the beginning of the plasma column will have expanded further than the younger electrons right behind the laser pulse, which leads to a conical profile for the expanding shell. The consequence of the conical profile is that the electron expansion is axisymmetric: At each moment in time every radial component of the electric field around the circumference of the plasma column is opposed by another component of equal magnitude on the opposite side. Therefore, the accompanying radial current produces no radiation that can propagate to the far field, even though the electrons experience acceleration in the radial direction over the rise time of the expansion wave. However, there is no such symmetry in the longitudinal direction. The rising and falling portions of the wave, that is, the initial expulsion and subsequent relaxation of electrons from the plasma column surface, are temporally and spatially nonuniform.

This asymmetry will thus drive a pulse of longitudinal surface current in the direction of laser propagation and this generates the broadband microwave signal. The longitudinal current pulse will be modulated over the length of the plasma column by the varying intensity of the laser pulse as it focuses and diffracts and this in turn modifies the waveform of the resulting microwave field. The current modulation is what we believe causes the spectral shift we note in Fig. 5 as the spatial profile of the plasma varies with the input laser pulse energy. 

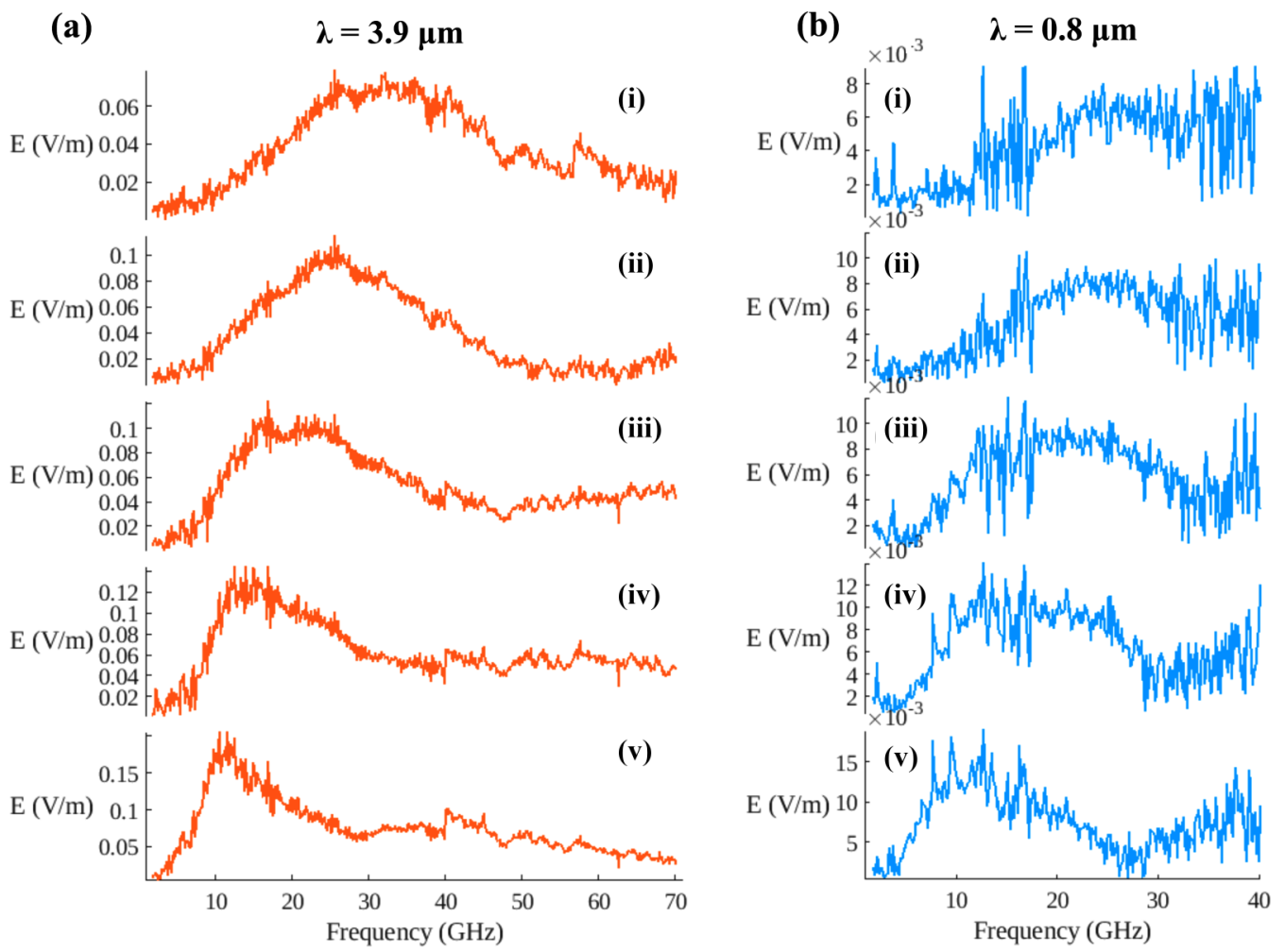

FIG. 5. Calibrated spectra of the free-space microwave electric field radiating from the air plasmas as a function of laser pulse energy. The antennas are positioned at $\theta=90^{\circ}$ relative to the laser propagation axis. The two columns of spectra correspond to the different lasers with (a) $\lambda_{0}=3.9 \mu \mathrm{m}$ and (b) $\lambda_{0}=0.8 \mu \mathrm{m}$. The subplots within (a) and (b) are numbered (i)-(v), corresponding to laser pulse energies of 2, 3, 5, 7.5 , and $12 \mathrm{~mJ}$, respectively. The spectra in (b) have been truncated at $40 \mathrm{GHz}$ because the noise rises above the signal. The noise amplitude increases linearly with frequency when the calibration factors are applied. The signal-to-noise ratio in (a) is much larger and therefore the spectra do not have this limitation.

Additional data linking the current modulation to the plasma shape is given in Sec. 3 in [19].

Figure 6 illustrates the process. On the macroscopic level, which is one where the total length of the plasma is the relevant length scale, the longitudinal current increases and decreases in magnitude with the amplitude of the electron expansion wave that travels coherently behind the laser pulse. For longitudinally short plasmas generated under tight focusing conditions such as in these experiments, we find that this current modulation significantly influences the resulting microwave spectrum within the $2-70 \mathrm{GHz}$ bandwidth of our measurements. On the other hand, we have observed in other experiments conducted in a limited bandwidth of $2-14 \mathrm{GHz}$ with longitudinally extended plasmas where nonlinear propagation of the laser is more pronounced that the microwave spectrum changes weakly with the plasma length [12]. The qualitative difference of these observations arises from the dependence of the coherent buildup of the microwaves on the plasma shape and is discussed further in Sec. 3 in [19].

A prior experiment performed by Proulx et al. [39] also implies an amplitude modulation of the electron expansion wave over the plasma length. Near-field measurements of radio-frequency voltage waveforms induced on the exposed end of a coaxial cable held $0.5 \mathrm{~cm}$ from an approximately 20-cm-long filament-produced plasma increased in amplitude, then diminished, and reversed polarity as the antenna translated longitudinally. While the receiver's $1-\mathrm{GHz}$ maximum frequency would have significantly filtered the time evolution of the electric field, it is likely that the near-field measurements and the radiation we have measured are aspects of the same phenomenon. The reported characteristic length scale of the longitudinal variation of the near-field amplitude approaches the plasma length, which agrees with our description of how the electron expansion wave varies slowly in amplitude on the macroscopic scale.

On the microscopic scale (i.e., the bottom of Fig. 6) we are concerned with the electron trajectories in and immediately following the laser pulse. For a given intensity, we expect that the $\lambda_{0}^{2}$ dependence of the residual energy causes the conical electron expansion wave to carry a larger radial current. Since the amplitude of the longitudinal surface current is determined by that of the radial expansion wave, the microwave radiation power should increase with laser wavelength, which is what we have quantified in Figs. 2-5. The theory and simulations of the microwave generation mechanism that capture these microscopic and macroscopic scales of the plasma dynamics are reported in [40].

\section{B. Laser propagation simulations}

To further support the idea that the $\lambda_{0}^{2}$ wavelength scaling of the residual energy is what drives the difference in microwave power between the MIR and NIR laser pulses, we perform simulations of the laser propagation to estimate the 


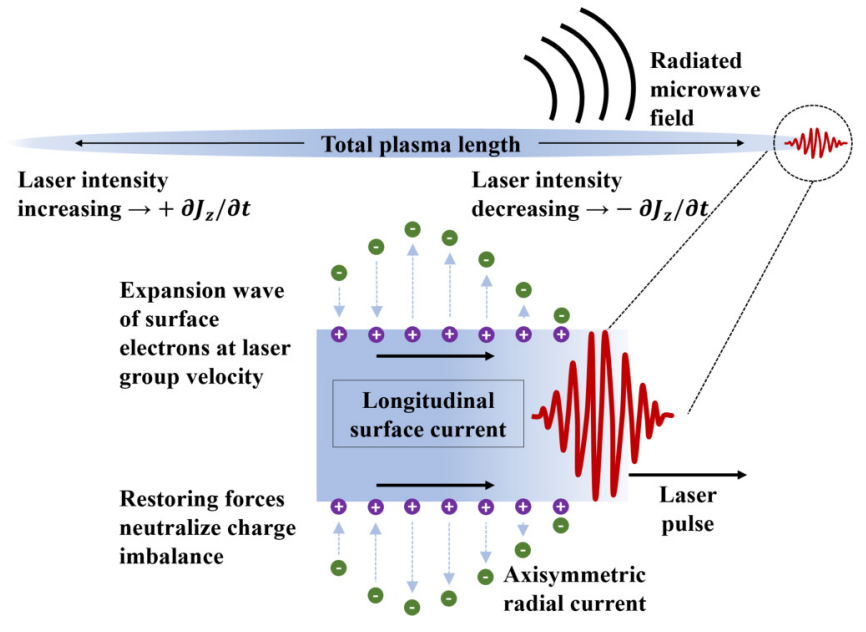

FIG. 6. Diagram describing the microwave radiation mechanism. The laser pulse propagates from left to right. The top portion of the figure shows the macroscopic picture of the slowly varying laser intensity and plasma properties that produce a current modulation on microwave timescales. The dashed lines indicate what occurs on a microscopic scale in the vicinity of the laser pulse as it propagates. The vertical arrows extending radially from the plasma point in the direction of the radial electron velocity. While it is initially outward, electrostatic attraction from the plasma draws the electrons back, indicated by the change in the arrows' direction. Collisions with neutrals serve as an additional restoring force that ultimately neutralizes the charge imbalance.

typical electron density and laser fluence of the experiments. The purpose of these simulations is to point out that the MIR laser plasma generates greater microwave power in spite of the fact that the peak intensity (see Sec. 4 in [19]) of the MIR pulses is predicted to be half that of the NIR pulses and the number of available charge carriers is almost 100 times smaller. Therefore, the residual electron energy must largely account for microwave signals we measure.

The simulations use the propagation code SeaRay [41], which solves the unidirectional pulse propagation equation [42] in cylindrical symmetry. They account for the nonlinear air response due to the Kerr effect, ionization, and group velocity dispersion. SeaRay cannot simulate the plasma dynamics, but it does calculate the electron density based on the tunneling ionization rate given by the Perelomov-PopovTerent'ev (PPT) model [43].

The input pulses are transform limited and Gaussian in space and time (with durations of 50 fs for the NIR pulses and 100 fs for the MIR pulses) having phase fronts initialized with only the $f / 15$ focusing of the OAP mirror. The Gaussian envelope profile and initial phase front curvature are approximations that simplify the simulations and do not reflect the detailed structure of the intensity and phase of the input pulses in the experiments. However, the difference in wavelength ultimately dominates the plasma generation. The radial spatial increment is $\Delta r=30 \mu \mathrm{m}$, while the time step is sufficiently small to resolve the carrier frequency determined by each value of $\lambda_{0}$. The fluence and electron density are stored in longitudinal increments of $\Delta z=500 \mu \mathrm{m}$, although the simulation adaptively increments the longitudinal step while running. We used values for the nonlinear refractive index of $n_{2}=3.2 \times 10^{-19} \mathrm{~cm}^{2} / \mathrm{W}$ for $\lambda_{0}=0.8 \mu \mathrm{m} \mathrm{[44]} \mathrm{and}$ $n_{2}=7.6 \times 10^{-20} \mathrm{~cm}^{2} / \mathrm{W}$ for $\lambda_{0}=3.9 \mu \mathrm{m}$ [45]. We assume that the pulses ionize molecular oxygen, which is the dominant source of free electrons in air plasmas generated by strong laser fields [46], owing to its lower ionization potential of $12.1 \mathrm{eV}$ versus $15.6 \mathrm{eV}$ for molecular nitrogen. The simulations were performed over a range of input pulse energies that overlaps that of the experiments.

Figure 7 maps the laser fluence and electron density for 5-mJ NIR and MIR pulses as a function of propagation distance and radius, where $r=0$ is the laser axis and $z=0$ is the location of the geometric focus. The simulations show that the plasma causes the pulses to start defocusing before the geometric focus, which is expected because its refractive index is less than unity. The pulse evolution is more complicated in the NIR case, since it is above the self-focusing critical power and therefore elicits a significant nonlinear response of the air. The ratio of input to critical power is $P / P_{\mathrm{cr}} \sim 10$, where $P / P_{\mathrm{cr}}=1$ is the self-focusing threshold; $P_{\mathrm{cr}} \propto \lambda_{0}^{2} / n_{2}$, where $n_{2}$ is the nonlinear refractive index. The MIR pulse power is well below the threshold $\left(P / P_{\mathrm{cr}} \sim 0.2\right)$ and does not exhibit significant nonlinear propagation.

Axial and radial lineouts of the laser fluence and electron density are given in Figs. 7(c) and 7(f). Quantitatively, the on-axis laser fluence is similar between the NIR and MIR cases, although abrupt jumps in the fluence and electron density that accompany nonlinear collapse of the NIR pulse are clear in Fig. 7(c). Comparison of the electron densities in the maps and lineouts shows that the NIR pulses generate a plasma that has about 100 times greater electron density than the MIR laser plasma. The NIR simulations predict detailed structure of the electron density due to the nonlinear propagation in Figs. 7(c) and 7(f) that might result in complicated plasma dynamics after the laser pulse passes. It is not clear from our experimental microwave data whether this affects the radiation source, which is the diffusive expansion wave driving the longitudinal current pulse at the plasma surface.

In light of the microwave generation mechanism proposed above, we can make a few observations relating to the microwave radiation even though there are no plasma dynamics in the simulations. Bear in mind that the plots of the simulation data represent a time-integrated picture of the laser propagation and ionization and do not include, for example, extinction of the plasma by recombination. The plasma size estimated as a fraction of the peak on-axis density appears to be about the same between the MIR and NIR cases. Figures 7(b), 7(e), and 7(f) indicate that the maximum plasma radius is about $150 \mu \mathrm{m}$ for both wavelengths. The cross-sectional area of plasma through which the microwaveproducing currents flow $\left(I=\int \mathbf{J} \cdot d \mathbf{A}\right.$ ) will contribute to the radiation amplitude, and since the plasma sizes are rather similar, we cannot completely attribute the factor of $\sim 100$ difference in the experimentally measured microwave power to the wavelength dependence of the plasma size. The simulations together with our experimental data imply that if the MIR and NIR laser plasmas are about the same size and the MIR plasma has orders of magnitude less bulk electron density, then the kinetic energy of the electrons is likely the 

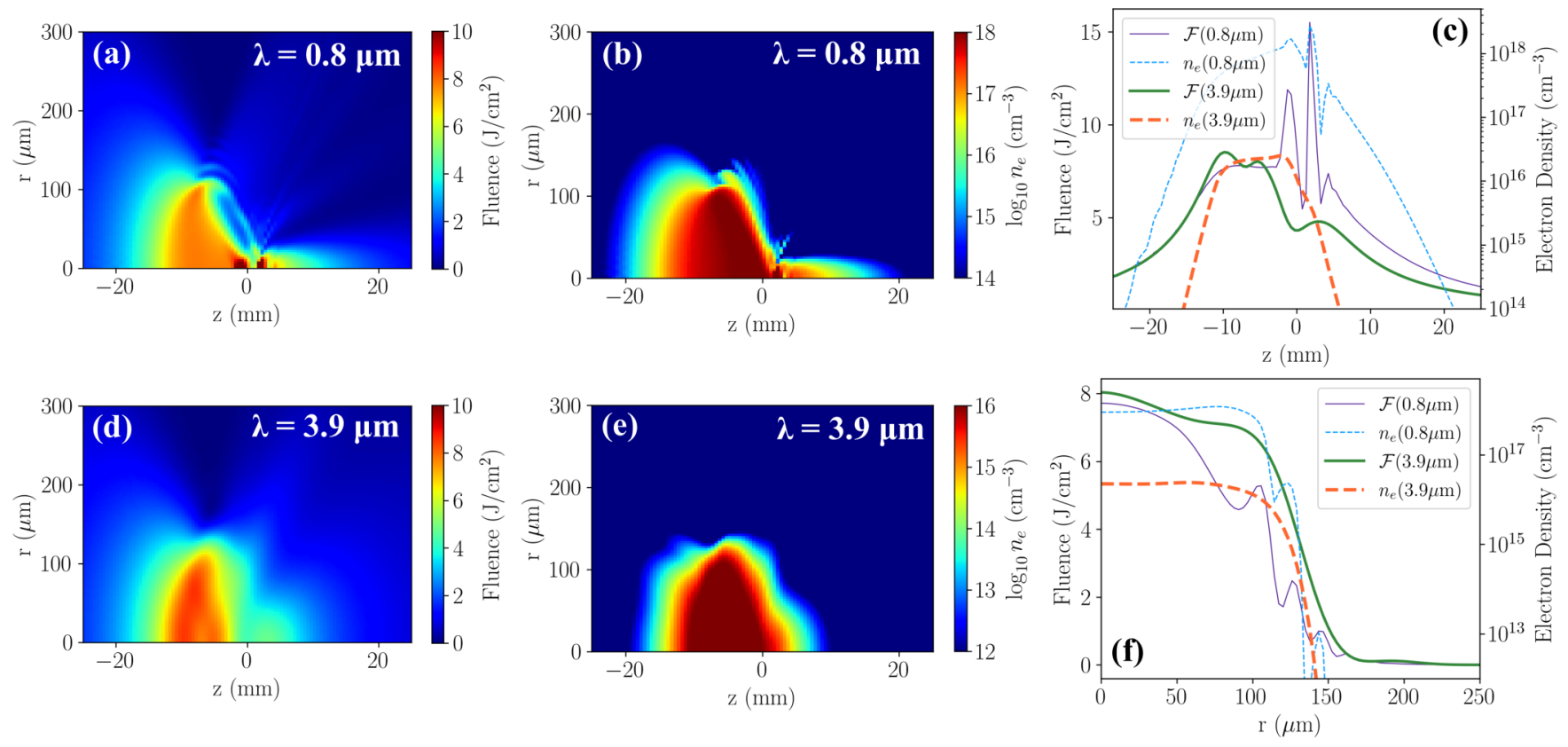

FIG. 7. Simulated laser fluence and electron density maps for 5-mJ input pulses focused at $f / 15$. (a) Fluence and (b) electron density for $\lambda_{0}=0.8 \mu \mathrm{m}$ at 50-fs pulse duration. (d) Fluence and (e) electron density for $\lambda_{0}=3.9 \mu \mathrm{m}$ at 100 -fs pulse duration. Note the difference in the electron density color bar limits for (b) versus (e). (c) Axial lineouts $(r=0)$ of the fluence $\mathcal{F}$ and electron density $n_{e}$ for both laser wavelengths. (f) Radial lineouts at $z=-5 \mathrm{~mm}$, which is where the plasma has the greatest radial extent. The thin solid and dashed lines correspond to fluence and electron density for $\lambda_{0}=0.8 \mu \mathrm{m}$, respectively, while the heavy solid and dashed lines correspond to $\lambda_{0}=3.9 \mu \mathrm{m}$. The longitudinal position $z=0$ is at the geometric focal plane of the focusing optic.

primary factor causing the difference we measure in the microwave radiation power.

\section{CONCLUSION}

Our experiments show that we are able to control the amplitude and frequency content of broadband microwave pulses generated by focusing ultrashort laser pulses in air through the laser wavelength and pulse energy. These experiments establish the laser wavelength dependence of the microwave generation, while the wavelength scaling of the $\mathrm{THz}$ generation has been explored previously in simulations and experiments [47-49]. Whether or not the increase in the microwave power as a function laser wavelength holds continuously over large ranges of $\lambda_{0}$ is not known, as many factors such as molecular absorption of the laser by air constituents will affect the ionization and laser propagation [50]. While we used two different lasers to access the NIR and MIR regimes, wavelength tunable OPA-based ultrashort pulse lasers with millijoule pulse energies are proliferating, which would allow for tuning of the microwaves without needing wholly different laser infrastructure. The air plasmas may prove to be useful sources of microwave radiation for ultrabroadband single-shot and pump-probe spectroscopy for measuring material prop- erties in a frequency regime that is traditionally difficult to access, but of growing technological significance.

In addition to demonstrating control of the secondary radiation, we proposed a generation mechanism that explains our experimental results and is supported by modeling of the laser propagation and plasma generation. Our findings validate the notion that the electron residual energy ultimately drives the plasma currents that produce the microwaves. Direct simulation of the microwave generation across the multiple relevant temporal and spatial scales is left for future research.

\section{ACKNOWLEDGMENTS}

A.E. thanks Hugh Pohle for assistance with performing the antenna gain measurements for the receiver calibrations. This material is based upon work supported by the Air Force Office of Scientific Research under Awards No. FA9550-16RDCOR325, No. FA9550-19RDCOR027, No. FA9550-16-10121, and No. FA9550-16-10259. The research was performed while R.P. held a National Research Council (NRC) Research Associateship Award at AFRL. D.W. acknowledges support from the DOE NNSA SSGF program under Award No. DE-NA0003864. A.E. acknowledges support from the U.S. Naval Research Laboratory through the Jerome and Isabella Karle Postdoctoral Fellowship.
[1] B. Miao, L. Feder, J. Elle, A. J. Goers, D. Woodbury, F. Salehi, J. K. Wahlstrand, and H. M. Milchberg, Coherent ultrabroadband laser-assisted injection radiation from a laser plasma accelerator, Phys. Rev. E 98, 043206 (2018).
[2] T. Matsuoka, C. McGuffey, P. G. Cummings, Y. Horovitz, F. Dollar, V. Chvykov, G. Kalintchenko, P. Rousseau, V. Yanovsky, S. S. Bulanov, A. G. R. Thomas, A. Maksimchuk, and K. Krushelnick, Stimulated Raman Side Scattering in 
Laser Wakefield Acceleration, Phys. Rev. Lett. 105, 034801 (2010).

[3] A. Rundquist, C. G. Durfee, Z. Chang, C. Herne, S. Backus, M. M. Murnane, and H. C. Kapteyn, Phase-matched generation of coherent soft X-rays, Science 280, 1412 (1998).

[4] K. Y. Kim, J. H. Glownia, A. J. Taylor, and G. Rodriguez, Terahertz emission from ultrafast ionizing air in symmetry-broken laser fields, Opt. Express 15, 4577 (2007).

[5] X. Xie, J. Dai, and X.-C. Zhang, Coherent Control of THz Wave Generation in Ambient Air, Phys. Rev. Lett. 96, 075005 (2006).

[6] K. Y. Kim, A. J. Taylor, J. H. Glownia, and G. Rodriguez, Coherent control of terahertz supercontinuum generation in ultrafast laser-gas interactions, Nat. Photon. 2, 605 (2008).

[7] V. A. Andreeva, O. G. Kosareva, N. A. Panov, D. E. Shipilo, P. M. Solyankin, M. N. Esaulkov, P. González de Alaiza Martínez, A. P. Shkurinov, V. A. Makarov, L. Bergé, and S. L. Chin, Ultrabroad Terahertz Spectrum Generation from an Air-Based Filament Plasma, Phys. Rev. Lett. 116, 063902 (2016).

[8] D. Jang, R. M. Schwartz, D. Woodbury, J. Griff-McMahon, A. H. Younis, H. M. Milchberg, and K.-Y. Kim, Efficient terahertz and Brunel harmonic generation from air plasma via mid-infrared coherent control, Optica 6, 1338 (2019).

[9] A. D. Koulouklidis, C. Gollner, V. Shumakova, V. Y. Fedorov, A. Pugžlys, A. Baltuška, and S. Tzortzakis, Observation of extremely efficient terahertz generation from mid-infrared twocolor laser filaments, Nat. Commun. 11, 292 (2020).

[10] A. Englesbe, J. Elle, R. Reid, A. Lucero, H. Pohle, M. Domonkos, S. Kalmykov, K. Krushelnick, and A. SchmittSody, Gas pressure dependence of microwave pulses generated by laser-produced filament plasmas, Opt. Lett. 43, 4953 (2018).

[11] A. V. Mitrofanov, D. A. Sidorov-Biryukov, M. M. Nazarov, A. A. Voronin, M. V. Rozhko, A. B. Fedotov, and A. M. Zheltikov, Coherently enhanced microwave pulses from midinfrared-driven laser plasmas, Opt. Lett. 46, 1081 (2021).

[12] A. Janicek, E. Thornton, T. Garrett, A. Englesbe, J. Elle, and A. Schmitt-Sody, Length dependence on broadband microwave emission from laser-generated plasmas, IEEE Trans. Plasma Sci. 48, 1979 (2020).

[13] S. Tzortzakis, G. Méchain, G. Patalano, Y.-B. André, B. Prade, M. Franco, A. Mysyrowicz, J.-M. Munier, M. Gheudin, G. Beaudin, and P. Encrenaz, Coherent subterahertz radiation from femtosecond infrared filaments in air, Opt. Lett. 27, 1944 (2002).

[14] C. D'Amico, A. Houard, M. Franco, B. Prade, A. Mysyrowicz, A. Couairon, and V. T. Tikhonchuk, Conical Forward THz Emission from Femtosecond-Laser-Beam Filamentation in Air, Phys. Rev. Lett. 98, 235002 (2007).

[15] P. Agostini and L. F. DiMauro, Atoms in high intensity midinfrared pulses, Contemp. Phys. 49, 179 (2008).

[16] N. E. Andreev, M. E. Veisman, S. P. Goreslavskii, and M. V. Chegotov, Residual electron momentum and energy in a gas ionized by a short high-power laser pulse, Plasma Phys. Rep. 27, 278 (2001).

[17] P. Pulsifer, J. P. Apruzese, J. Davis, and P. Kepple, Residual energy and its effect on gain in a Lyman- $\alpha$ laser, Phys. Rev. A 49, 3958 (1994).

[18] B. M. Penetrante and J. N. Bardsley, Residual energy in plasmas produced by intense subpicosecond lasers, Phys. Rev. A 43, 3100 (1991).
[19] See Supplemental Material at http://link.aps.org/supplemental/ 10.1103/PhysRevA.104.013107 for additional context regarding the microwave generation mechanism, and ancillary results of the experiments and simulations.

[20] D. Marpaung, J. Yao, and J. Capmany, Integrated microwave photonics, Nat. Photon. 13, 80 (2019).

[21] D. H. Auston and P. R. Smith, Generation and detection of millimeter waves by picosecond photoconductivity, Appl. Phys. Lett. 43, 631 (1983).

[22] F. Sabath and E. Mokole, Ultra-Wideband, Short-Pulse Electromagnetics 10 (Springer, Berlin, 2014).

[23] H. Aggrawal, P. Chen, M. M. Assefzadeh, B. Jamali, and A. Babakhani, Gone in a picosecond: Techniques for the generation and detection of picosecond pulses and their applications, IEEE Microwave Mag. 17, 24 (2016).

[24] J. S. Pearlman and G. H. Dahlbacka, Emission of rf radiation from laser-produced plasmas, J. Appl. Phys. 49, 457 (1978).

[25] J. A. Aspiotis, N. Barbieri, R. Bernath, C. G. Brown, M. Richardson, and B. Y. Cooper, in Enabling Technologies and Design of Nonlethal Weapons, edited by G. T. Shwaery, J. G. Blitch, and C. Land, SPIE Proc. Vol. 6219 (SPIE, Bellingham, 2006), pp. 56-63.

[26] S. Varma, J. Spicer, B. Brawley, and J. A. Miragliotta, Plasma enhancement of femtosecond laser-induced electromagnetic pulses at metal and dielectric surfaces, Opt. Eng. 53, 051515 (2014).

[27] F. Consoli, V. T. Tikhonchuk, M. Bardon, P. Bradford, D. C. Carroll, J. Cikhardt, M. Cipriani, R. J. Clarke, T. E. Cowan, C. N. Danson et al., Laser produced electromagnetic pulses: Generation, detection and mitigation, High Power Laser Sci. Eng. 8, E22 (2020).

[28] J.-L. Dubois, F. Lubrano-Lavaderci, D. Raffestin, J. Ribolzi, J. Gazave, A. Compant La Fontaine, E. d'Humières, S. Hulin, P. Nicolaï, A. Poyé, and V. T. Tikhonchuk, Target charging in short-pulse-laser-plasma experiments, Phys. Rev. E 89, 013102 (2014).

[29] A. Davidson, G. M. Petrov, B. Rock, P. Grugan, D. Gordon, B. Hafizi, A. Ting, and J. Peñano, Dynamic sheath formation and sub-THz radiation from laser-metal interactions, Phys. Plasmas 27, 073101 (2020).

[30] G. Andriukaitis, T. Balčiūnas, S. Ališauskas, A. Pugžlys, A. Baltuška, T. Popmintchev, M.-C. Chen, M. M. Murnane, and H. C. Kapteyn, $90 \mathrm{GW}$ peak power few-cycle mid-infrared pulses from an optical parametric amplifier, Opt. Lett. 36, 2755 (2011).

[31] C. A. Balanis, Antenna Theory: Analysis and Design (Wiley, New York, 2016).

[32] E. Muehldorf, The phase center of horn antennas, IEEE Trans. Antennas Propag. 18, 753 (1970).

[33] J. D. Jackson, Classical Electrodynamics (Wiley, New York, 1998).

[34] P. Sprangle, J. R. Peñano, B. Hafizi, and C. A. Kapetanakos, Ultrashort laser pulses and electromagnetic pulse generation in air and on dielectric surfaces, Phys. Rev. E 69, 066415 (2004).

[35] C. D'Amico, A. Houard, S. Akturk, Y. Liu, J. L. Bloas, M. Franco, B. Prade, A. Couairon, V. T. Tikhonchuk, and A. Mysyrowicz, Forward $\mathrm{THz}$ radiation emission by femtosecond filamentation in gases: Theory and experiment, New J. Phys. 10, 013015 (2008). 
[36] S. Kalmykov, J. Elle, and A. Schmitt-Sody, Radiation emission at Langmuir frequency from laser wake in longitudinally stratified plasma column, Plasma Phys. Control. Fusion 62, 115022 (2020).

[37] S. Kalmykov, J. Elle, and A. Schmitt-Sody, Reversal of laser wake phase velocity generates high-power broadband Cherenkov signal, Plasma Phys. Control. Fusion 63, 045024 (2021).

[38] A. A. Voronin and A. M. Zheltikov, Laser-driven tunneling photocurrent as a source of midinfrared to microwave multidecade supercontinua yoked to high-order harmonics, Phys. Rev. A 101, 043813 (2020).

[39] A. Proulx, A. Talebpour, S. Petit, and S. Chin, Fast pulsed electric field created from the self-generated filament of a femtosecond Ti:sapphire laser pulse in air, Opt. Commun. 174, 305 (2000).

[40] T. Garrett, J. Elle, M. White, R. Reid, A. Englesbe, R. Phillips, P. Mardahl, A. Schmitt-Sody, E. Thornton, J. Wymer, A. Janicek, and O. Sale, Generation of RF by atmospheric filaments, arXiv:2102.12657.

[41] D. Gordon, USNavalResearchLaboratory: SeaRay, Zenodo (2020).

[42] M. Kolesik, J. V. Moloney, and M. Mlejnek, Unidirectional Optical Pulse Propagation Equation, Phys. Rev. Lett. 89, 283902 (2002).

[43] V. S. Popov, Tunnel and multiphoton ionization of atoms and ions in a strong laser field (Keldysh theory), Phys. Usp. 47, 855 (2004).
[44] A. Couairon and A. Mysyrowicz, Femtosecond filamentation in transparent media, Phys. Rep. 441, 47 (2007).

[45] S. Zahedpour, S. W. Hancock, and H. M. Milchberg, Ultrashort infrared 2.5-11 $\mu \mathrm{m}$ pulses: Spatiotemporal profiles and absolute nonlinear response of air constituents, Opt. Lett. 44, 843 (2019).

[46] A. Sharma, M. N. Slipchenko, M. N. Shneider, X. Wang, K. A. Rahman, and A. Shashurin, Counting the electrons in a multiphoton ionization by elastic scattering of microwaves, Sci. Rep. 8, 2874 (2018).

[47] A. Nguyen, K. J. Kaltenecker, J.-C. Delagnes, B. Zhou, E. Cormier, N. Fedorov, R. Bouillaud, D. Descamps, I. Thiele, S. Skupin, P. U. Jepsen, and L. Bergé, Wavelength scaling of terahertz pulse energies delivered by two-color air plasmas, Opt. Lett. 44, 1488 (2019).

[48] V. Y. Fedorov and S. Tzortzakis, Extreme THz fields from twocolor filamentation of midinfrared laser pulses, Phys. Rev. A 97, 063842 (2018).

[49] M. Clerici, M. Peccianti, B. E. Schmidt, L. Caspani, M. Shalaby, M. Giguère, A. Lotti, A. Couairon, F. Légaré, T. Ozaki, D. Faccio, and R. Morandotti, Wavelength Scaling of Terahertz Generation by Gas Ionization, Phys. Rev. Lett. 110, 253901 (2013).

[50] V. Shumakova, S. Ališauskas, P. Malevich, C. Gollner, A. Baltuška, D. Khartashov, A. Zheltikov, A. Mitrofanov, A. Voronin, D. Sidorov-Biryukov, and A. Pugžlys, Filamentation of mid-IR pulses in ambient air in the vicinity of molecular resonances, Opt. Lett. 43, 2185 (2018). 